\title{
Implementação de um módulo de circulação através do software de funções integradas VIRTYA/VTLS : a experiência do Sistema de Bibliotecas da UNICAMP
}

\author{
Ademir Giacomo Pietrosanto \\ Universidade Estadual de Campinas \\ Instituto de Economia \\ Campinas - SP - Brasil \\ e-mail: pietro@eco.unicamp.br \\ Gildenir Carolino Santos \\ Universidade Estadual de Campinas \\ Faculdade de Educação \\ Campinas - SP - Brasil \\ e-mail: gilbfe@unicamp.br \\ Márcia Aparecida D'Áloia Pillon \\ Universidade Estadual de Campinas \\ Biblioteca Central \\ Campinas - SP - Brasil \\ e-mail: biatende@unicamp.br

\section{Célia Aparecida Rodrigues} \\ Universidade Estadual de Campinas \\ Instituto de Estudos da Linguagem \\ Campinas - SP - Brasil \\ e-mail: celia-ar@iel.unicamp.br
}

\section{Resumo}

$\mathrm{O}$ artigo apresenta o resultado de uma experiência realizada no Sistema de Bibliotecas da UNICAMP (SBU) sobre a implantação do módulo Circulação do Software de funções integradas VIRTUA/VTLS a partir do estudo do formato USMARC aplicado a este módulo. O grupo de estudo do módulo Circulação, formado por bibliotecários do SBU, procura mostrar os métodos que foram adotados para a análise, definição e parametrização do referido módulo. Além disso, destaca as vantagens de utilização de um software de funções integradas voltado para o controle de materiais bibliográficos.

\author{
Palavras-chave \\ Bibliotecas-Circulação-Automação; \\ Formato bibliográfico USMARC; \\ VIRTUA-Software de funções integradas
}

\begin{abstract}
The article presents the result of an experience accomplished in the System of Libraries of UNICAMP (SBU) on the implantation of the module Circulation of the Software of integrated functions VIRTUA/VTLS starting from the study of the format USMARC applied to this module. The group of study of the module Circulation, formed by librarians of SBU, tries to show the methods that were adopted for the analysis, definition and parameterization of the referred module. Besides, it highlights the advantages of use of a software of integrated functions gone back to the control of bibliographical materials.
\end{abstract}

\section{Key-words}

Libraries-Circulation-Automation; USMARC bibliographic format; Software of integrated functions VIRTUA

${ }^{1}$ A presentado inicialmente no XI SN BU - 24 a 28 abr. 2000 - Florianópol is, SC. 


\section{INTRODUÇÃO}

O processo de informatização do controle de Circulação do Sistema de Bibliotecas da UNICAMP (SBU) data do início dos anos 90 .

A primeira biblioteca que implantou o controle de Circulação automatizado no SBU foi o Centro de Documentação "Lucas Gamboa" do Instituto de Economia (CEDOC/IE) que, à época, também iniciava a automação do seu acervo através da rede Bibliodata/CALCO. O embrião do empréstimo automatizado, trazido pelo bibliotecário do CEDOC/IE, fez com que as demais bibliotecas do SBU se interessassem em automatizar o controle de circulação, substituindo assim o controle manual (PIETROSANTO, SANTOS e MENDES, 1994).

Juntamente com o CEDOC/IE, também aderiram ao controle automatizado as Bibliotecas da Área de Engenharia (BAE), do Instituto de Filosofia e Ciências Humanas (BIFCH), da Faculdade de Educação (BFE), do Instituto de Física (BIF) e do Instituto de Química (BIQ).

Logo após a implantação da circulação automatizada nas bibliotecas, que também recebiam suporte para manutenção do software pelo Analista de Sistemas do CEDOC/IE, criava-se um grupo para analisar as necessidades advindas da utilização do programa.

Com a crescente demanda e ampliação do software, o controle e manutenção passavam-se para a Biblioteca Central e CCUEC.

\section{ESCOLHA DO SOFTWARE PARA AS FUNÇÕES INTEGRADAS}

O processo para escolha do novo software para as Bibliotecas da UNICAMP teve início em março de 1997, com a estruturação de Grupos de Estudos, por funções:

- Catalogação

- Periódicos

- Referência (OPAC)

- Circulação.

Além dos profissionais Bibliotecários, foram também convidados para participar do processo de seleção do novo software, Docentes e Técnicos do Centro de Computação da UNICAMP.

Durante todo o período de estudos foram pesquisados e solicitadas demonstrações de softwares com características de Gestão Integrada das Funções de uma Biblioteca.

Em 01 de setembro de 1997, foi realizada na Biblioteca Central a reunião final de escolha do Software Integrado de Funções, tendo sido indicado o VIRTUA da VTLS Inc (USA).

\section{PLANO DE IMPLEMENTAÇÃO DO VIRTUA}

Em sua primeira fase do plano de implementação do VIRTUA foram ministrados os seguintes treinamentos para as bibliotecas do SBU:

FIT ANALYSIS (análise preliminar, para definição dos parâmetros do sistema); treinamento nas funções: OPAC , Catalogação e Circulação palestra com definições do Sistema ; palestra sobre Formatos MARC;

Treinamento de Windows95 para funcionários das Bibliotecas Setoriais - junho/98;

Treinamento na função OPAC para funcionários das Bibliotecas Setoriais - junho/98. 


\section{ESTRUTURA DO MÓDULO CIRCULAÇÃO DO VIRTUA}

No módulo Circulação, o VIRTUA controla todas as funções rotineiras, com base nas políticas de cada biblioteca, porém o SBU adotou uma política uniforme de prazos de empréstimo para todas as bibliotecas integrantes do Sistema. Desta forma, os funcionários autorizados podem executar funções de circulação em sua biblioteca que está integrada ao

SBU em rede, sem a necessidade de entrar num módulo específico de Circulação. O VIRTUA permite no módulo Circulação (VTLS, 1996):

\section{Empréstimos}

O software possibilita empréstimos com datas de devolução baseadas em características de itens e leitores, datas de devolução especificadas pelo operador e datas de devolução fixas. Todas as movimentações são efetuadas em tempo real. A tela do item exibe o status "Emprestado" e a data em que a devolução deve ser feita.

A seguir, apresenta-se a tela principal da modalidade "Empréstimo" :

Fig.1

\section{Tela de acesso ao módulo Empréstimo}

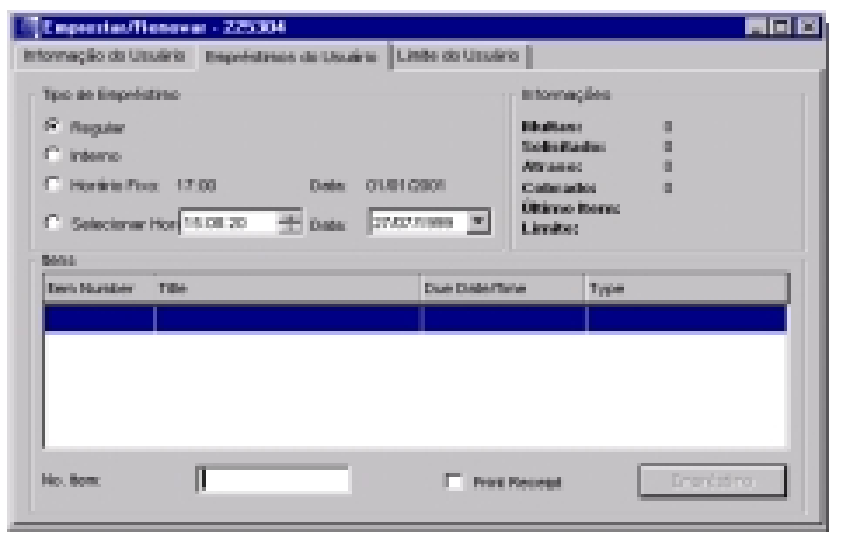

\section{Devoluções}

Neste item, o sistema verifica se o material está emprestado, informa se o usuário está em dia ou atrasado, calcula as multas que eventualmente devam ser impostas ao usuário e atribui os seguintes status: "Disponível", "Em transito", "Reservado" ao registro do item no OPAC. No caso de existirem solicitações não atendidas ou do item necessitar de encaminhamento a outras bibliotecas, o sistema atribui automaticamente o status

correspondente e alerta o operador de que é necessária uma administração especial do item. Faz também acompanhamento automático dos itens devolvidos em Bibliotecas diferentes da localização original.

Fig. 2

\section{Tela de acesso ao módulo Devolução}

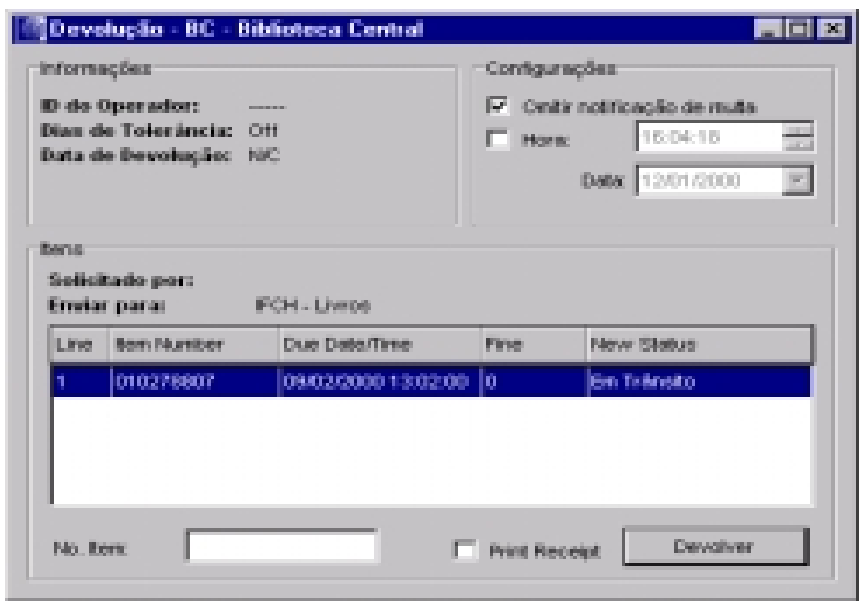

\section{Renovações}

O software permite a renovação de materiais ou de conjunto de materiais emprestados a um usuário. Nas renovações de um conjunto de materiais, o sistema gera notificações de renovação para informar o usuário das novas datas de devolução e dos materiais que não podem ser renovados por já haver 
solicitação de reserva para o mesmo. $\mathrm{O}$ sistema não permite a renovação de materiais com reservas ativas, com pedido de rechamada e nem com pedidos de empréstimos entre bibliotecas.

\section{Fig.3}

\section{Tela de acesso o módulo Renovação}

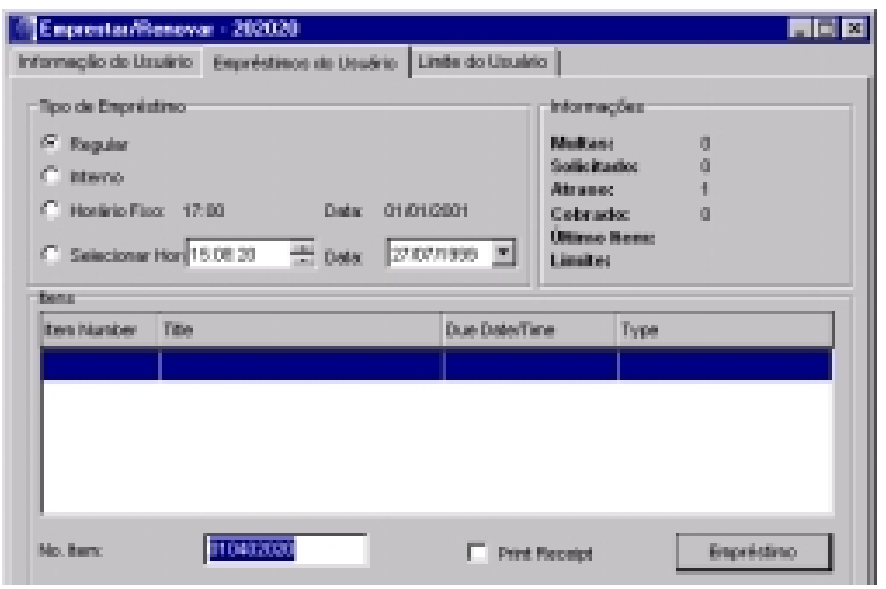

A biblioteca pode decidir que, tanto usuários, como funcionários, sejam autorizados a fazer reservas e pedidos de devolução dos materiais da coleção, ou restringir essas funções somente a funcionários. No caso de reserva, o sistema retém o material quando este é devolvido e gera uma notificação de reserva. Nos pedidos de devolução, o sistema gera uma notificação especial a ser enviada ao usuário que está com o material. Uma reserva feita para um material de empréstimo aberto gera, automaticamente, uma notificação de pedido de devolução. Os pedidos de reserva e devolução do VIRTUA especificam: o local de retirada do documento, se o leitor deseja uma cópia específica de um título ou se qualquer cópia o atenderá. A reserva pode ser feita pelo título do livro ou pelo número do item, em qualquer biblioteca do SBU, especificando-se o local de origem para a retirada do documento.
Fig. 4

Tela de acesso ao módulo Solicitação de Reservas

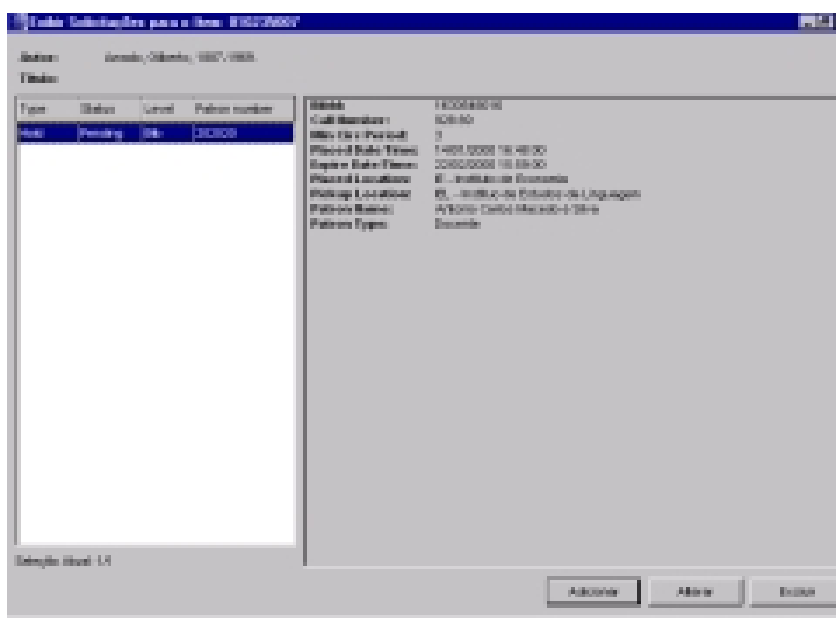

\section{Notificações de leitores}

O VIRTUA gera notificações de atraso, cartas de cobrança, faturas para reposição de materiais, cartas de renovação e pedidos de devolução, cujos textos são definidos pela biblioteca para os diversos tipos de usuários.

\section{Monitoramento da atividade dos usuários}

Para cada usuário, o VIRTUA inclui uma tela de Atividade do Usuário, cujo acesso é restrito ao pessoal autorizado. Essa tela fornece um resumo da atual situação do usuário junto ao sistema de Circulação, possibilitando a visualização dos pedidos de reservas ativas, de devolução e de empréstimo. Um registro de multas é anexado a cada tela de Atividade do Usuário. A biblioteca pode permitir que os próprios usuários consultem os registros de suas atividades mediante a entrada do número e do código especial do usuário (RA - Registro Acadêmico). 
Fig. 5

\section{Tela de acesso as informações do usuário quanto as suas atividades na biblioteca}

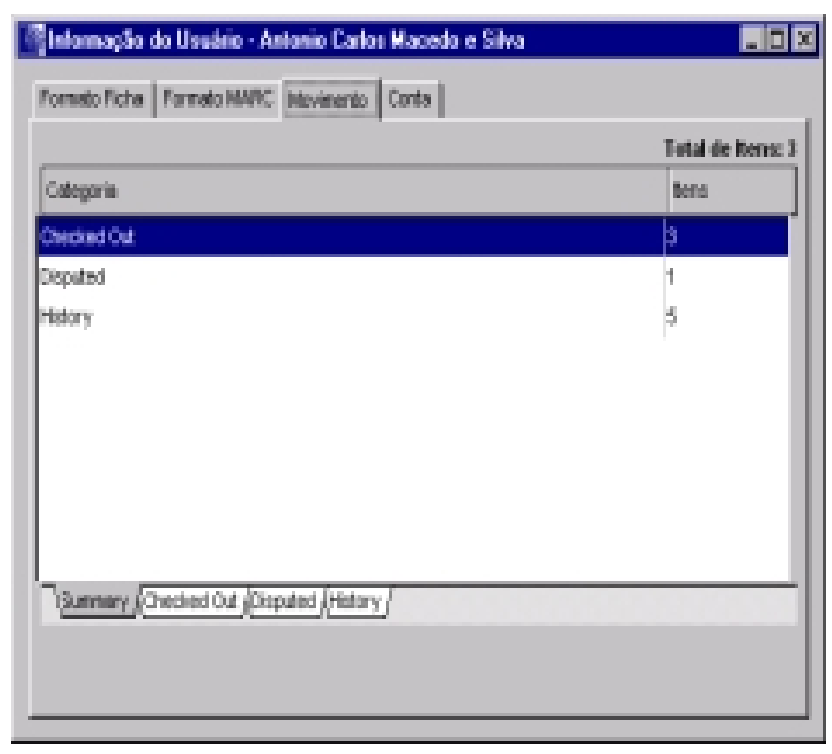

\section{Multas e taxas}

De acordo com as políticas estabelecidas pelo Sistema de Bibliotecas, o VIRTUA acompanha as multas devidas referentes a materiais em atraso. Visando ao máximo de flexibilidade, o sistema aceita diferentes valores de multa baseados na localização do material e no tipo de usuário. O Sistema permite também que se acrescentem as taxas aos registros de um usuário, além de aceitar pagamento integral, pagamento parcial ou exclusão das multas e taxas devidas pelo usuário. Além disso, o sistema bloqueia os usuários cujas dividas excedam o valor limite determinado pela biblioteca.

\section{Relatórios de controle de circulação}

O VIRTUA gera listas de pesquisas para livros desaparecidos e relatórios estatísticos que incluem os números de empréstimos, devoluções, multas impostas e cobradas ou perdoadas, reservas e pedidos de devolução. A estatística inclui empréstimos de renovações, todos classificados por ordem dos números de chamada.

\section{Bloqueio de usuários}

O VIRTUA bloqueia a retirada de materiais pelos usuários por diversos motivos, inclusive pela perda do documento de identidade e por multas não pagas. $\mathrm{O}$ sistema define códigos de bloqueio compatíveis com as infrações.

\section{Período de tolerância}

A biblioteca poderá estabelecer, dentro do próprio sistema, um período de tolerância para os materiais em atraso, no SBU ficou estabelecido em 03 (três) dias. Durante este período, serão cobradas multas sobre os materiais devolvidos. O sistema calcula as multas a partir da data de devolução ou do final do período de tolerância segundo a política estabelecida pelo SBU.

\section{Empréstimo entre bibliotecas}

Todas as bibliotecas do SBU poderão solicitar empréstimo entre as bibliotecas setoriais e também atender a outras instituições credenciadas no SBU.

\section{Controle de reserva}

O Controle de Reserva possibilita o empréstimo por hora ou apenas para o período noturno (overnight), a ser definido por cada biblioteca setorial, de determinados materiais indicados pelos docentes como bibliografia básica de cada disciplina e cuja utilização é restrita à consulta local. 


\section{MÉTODOS ADOTADOS NO SBU}

Os métodos que foram adotados para atender às necessidades do SBU, estão relacionados a seguir:

\section{a) Criação de Grupos de Estudos:}

Após a aquisição do software em meados de 1997, criaram-se os Grupos de Estudo das funções (Circulação, Catalogação, Aquisição e OPAC) para analisarem e implementarem os módulos no prazo máximo de 03 (três) anos.

\section{b) Tradução do manual:}

No módulo Circulação, preliminarmente o Grupo de Estudo teve como etapa principal a leitura e a tradução do manual da língua inglesa, para o português, tendo ocorrido modificações nas edições complementares enviadas pela VTLS Inc. para entendimento das funções.

\section{Seleção das categorias de usuários:}

Para facilitar a parametrização dos dados dos usuários no sistema VIRTUA, definiram-se as categorias de usuários juntamente com os devidos prazos de empréstimo.

\section{Itens bibliográficos cadastrados:}

Inicialmente, para qualidade e padronização dos itens bibliográficos, o software permite o cadastramento dos seguintes materiais:

- livros; dissertações e teses; periódicos (apenas o título da coleção).

- materiais que não circulam (obras de referência, coleção didática).

Além dos itens bibliográficos citados, também se encontram em cada acervo setorial, trabalho de conclusão de curso
(TCC), relatórios, folhetos, fitas de vídeo etc, e que serão avaliados, posteriormente, para inclusão dos mesmos no sistema, de forma padronizada.

\section{CATEGORIAS SELECIONADAS E PARAMETRIZADAS}

Uma das etapas da parametrização do software VIRTUA, foi a definição das categorias de usuários com seus respectivos prazos de empréstimo.

Discutiu-se a realidade de cada biblioteca setorial em relação às categorias a serem parametrizadas, que foram estabelecidas em número de 06 (seis). Estas estão relacionadas abaixo com o seu devido prazo de empréstimo (ATAS..., 1999):

TABELA 1

Parametrização das categorias de usuários e prazos de empréstimo

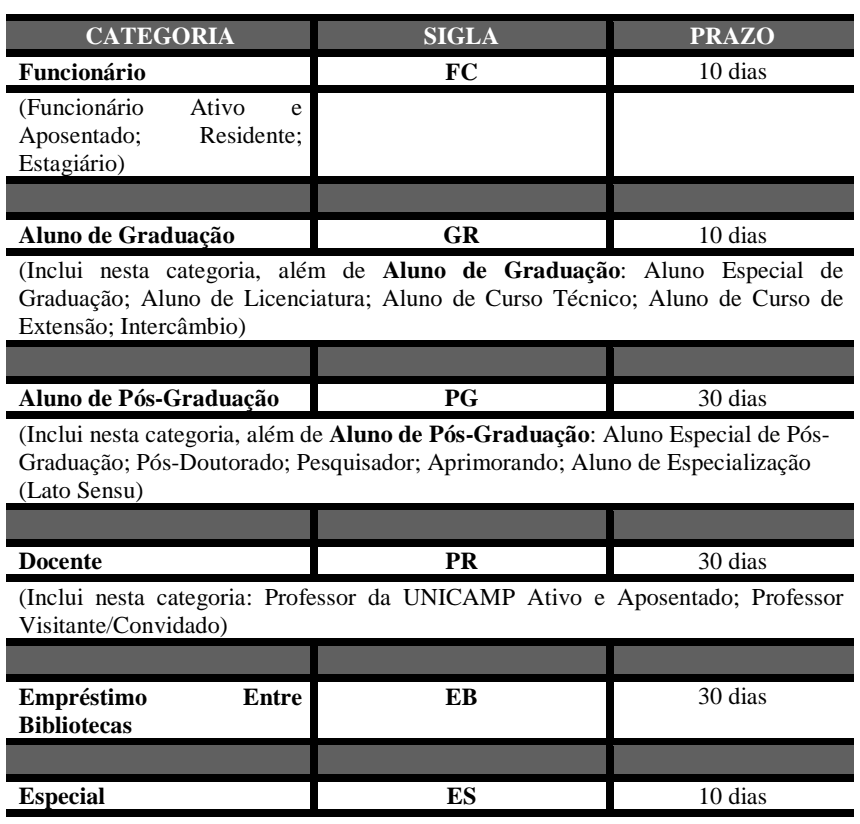

\section{CADASTRO DE USUÁRIOS}

Este item é um dos mais importantes do módulo Circulação, pois nele será colocada 
toda a informação do usuário, como pessoa física e/ou corporativa.

Existem vários campos a serem preenchidos, mas optou-se por um que forneça os dados principais do usuário.

Outro aspecto é que o formato apresentado tem padrão USMARC e integração direta com o módulo Catalogação, padronizandose desta forma a entrada de autoria.

A partir destes campos selecionados, desenvolveu-se a planilha para entrada de dados (workform) de usuários, demonstrada na Fig.6 e com as devidas descrições na Tabela 2, conforme seguem abaixo.

Fig.6

\begin{tabular}{l} 
Editor MARC do VIRTUA para \\
cadastramento de usuário \\
\hline
\end{tabular}

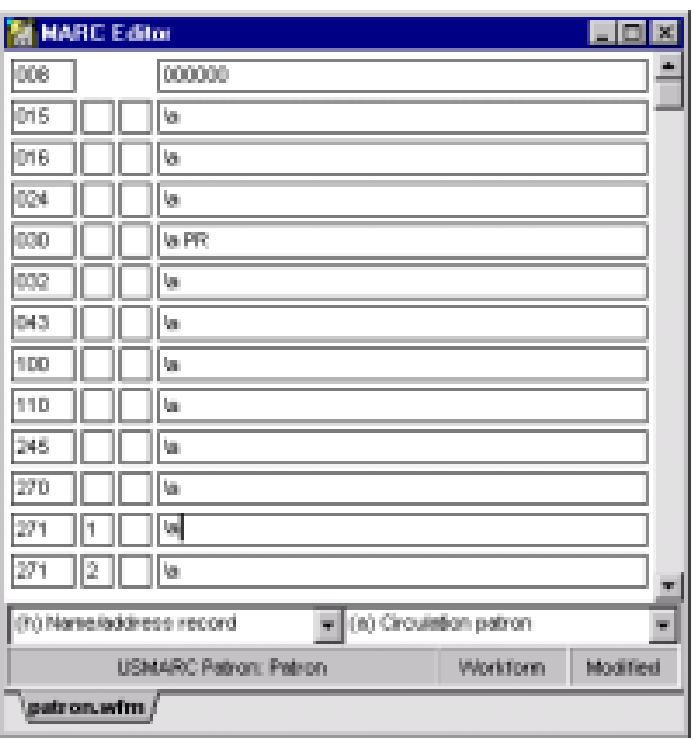

\section{TABELA 2}

\section{Descrição da planilha de cadastramento de usuário}

\begin{tabular}{|c|c|c|}
\hline $\begin{array}{l}\text { CAMPO } \\
\text { (TAG) }\end{array}$ & INDICADOR & DESCRIÇÃO \\
\hline 001 & & Controle de número gerado pelo sistema. \\
\hline 008 & & Campos fixos. \\
\hline 015 & & $\begin{array}{l}\text { Número do usuário : RA, RG (identificação do } \\
\text { usuário no sistema. Esse é o código principal a ser } \\
\text { usado }\end{array}$ \\
\hline 016 & & $\begin{array}{l}\text { Código alternativo (pode ser o RG, Título de } \\
\text { eleitor, passaporte, etc) também poderá ser usado } \\
\text { para empréstimo }\end{array}$ \\
\hline 030 & & $\begin{array}{l}\text { Tipo do usuário : professor, aluno, pesquisador, } \\
\text { etc. } \\
\text { Cadastra-se sempre com duas letras maiúsculas. } \\
\text { Ex.: PR (docente) }\end{array}$ \\
\hline 032 & & $\begin{array}{l}\text { Campo usado para gravar as transações do } \\
\text { usuário. Ex.: Multa. }\end{array}$ \\
\hline 043 & & $\begin{array}{l}\text { Usado para identificar o bloqueio do usuário. Ex.: } \\
\text { perda do cartão; danificação de material } \\
\text { bibliográfico, etc. }\end{array}$ \\
\hline 100 & & $\begin{array}{l}\text { Nome do usuário - Cadastra-se pelo nome e } \\
\text { sobrenome. }\end{array}$ \\
\hline 110 & & $\begin{array}{l}\text { Nome corporativo da biblioteca como usuário } \\
\text { (EEB). }\end{array}$ \\
\hline 245 & & $\begin{array}{l}\text { Tipo de usuário (como o campo } 30 \text { ) pode repetir } \\
\text { este campo. }\end{array}$ \\
\hline 270 & 1 & $\begin{array}{l}\text { endereço do usuário( o principal), tem vários } \\
\text { subcampos : } \\
\text { la = rua, avenida, numero, bairro, apto. bloco, etc.; } \\
\text { lb = cidade; } \\
\text { ld = Estado; le = código postal (CEP); lf = Pais; } \\
\text { l }=\text { telefone }\end{array}$ \\
\hline 270 & 2 & $\begin{array}{l}\text { Segundo endereço (repete todos os campos do } \\
\text { anterior). }\end{array}$ \\
\hline 271 & 1 & Endereço eletrônico = e-mail (principal). \\
\hline 271 & 2 & Endereço eletrônico = e-mail (alternativo). \\
\hline 301 & & $\begin{array}{l}\text { la }=\text { instituição a que pertence. Ex. Unicamp; } \backslash \mathrm{b}= \\
\text { Unidade. } \\
\text { Ex. Instituto de Economia; } \backslash \mathrm{d}=\text { Departamento. } \\
\text { Ex. CEDOC. }\end{array}$ \\
\hline 515 & & $\begin{array}{l}\text { Campo destinado para informações da conta do } \\
\text { usuário. Ex.: Multas. }\end{array}$ \\
\hline
\end{tabular}

\section{VANTAGENS DO SOFTWARE VIRTUA PARA O SBU}

O módulo Circulação tem as seguintes vantagens:

- interação e padronização com os outros módulos do sistema (OPAC, Catalogação, Periódicos) permitindo-se uma única entrada.

- funcionamento em rede. 
- bloqueio de usuários em uma única vez no sistema.

- permissão ao usuário para verificar o seu status junto ao empréstimo.

- permissão ao usuário para verificar localmente a sua situação junto ao SBU.

- emissão de relatórios para fins estatísticos.

- notificação da disponibilidade do item bibliográfico.

- permissão ao catalogador para a circulação em trânsito de material ainda não preparado (catalogação rápida - circ on the fly).

COMPARTILHAMENTO DO CONTROLE DE EMPRÉSTIMO VIA REDE

As unidades que antes mantinham o funcionamento do empréstimo local, não em rede, no momento da implantação do VIRTUA, beneficiaram-se com as vantagens da utilização em rede do sistema em tempo real. Isto favoreceu $o$ compartilhamento dos dados cadastrados em benefício principal aos usuários que se cadastravam em várias bibliotecas anteriormente, tornando inadequadas as rotinas do atendimento ao público.

\section{CONSIDERAÇÕES FINAIS}

O módulo de Circulação do VIRTUA atualmente se encontra em fase final de implementação. Decidiu-se que será feito em meados de fevereiro de 2000 um teste piloto em três bibliotecas do SBU para se verificar o funcionamento do software em relação ao cadastramento de usuários, empréstimo, renovação e devolução de itens bibliográficos e sua posterior utilização nas demais bibliotecas.
Além disso, com a aquisição do software, houve um grande avanço qualitativo no processo de automação do SBU.

Com as vantagens citadas, haverá condições reais de se monitorarem todas as atividades relacionadas ao empréstimo realizadas nas bibliotecas do SBU, facilitando assim, o controle de materiais bibliográficos e dos usuários via rede.

Outro grande fator que nos privilegia a implementar este software de funções integradas, é o fato de estarmos sendo a primeira instituição a utilizar o VIRTUA no Brasil.

\section{REFERÊNCIAS BIBLIOGRÁFICAS}

ATAS de reunião do Grupo de Estudo de Circulação - VIRTUA. Campinas, SP : [s.n.],1999.

PIETROSANTO, Ademir Giacomo, SANTOS, Gildenir Carolino, MENDES, Roberto Pedrozo. Sistema de controle de empréstimo automatizado nas bibliotecas da Universidade Estadual de Campinas - UNICAMP. In: SEMINÁRIO NACIONAL DE BIBLIOTECAS UNIVERSITÁRIAS, 8., 1994, Campinas, SP. Anais... Campinas, SP : UNICAMP/BC, 1994. p.286. (Resumo).

UNIVERSIDADE ESTADUAL DE CAMPINAS. Sistema de Bibliotecas. Implementação : definições do sistema VIRTUA. Campinas, SP. [citado: 1998]. [data de captura: 17/01/2000]. Apresentado em Power Point em 17/03/1998. Disponível na Internet: <http://www.unicamp.br/bc/client/sld001.htm>.

\section{UNIVERSIDADE ESTADUAL DE} CAMPINAS. Sistema de Bibliotecas. Plano de automação : modernização - software integrado de funções. Campinas, SP. [citado: 1998]. [data de captura: 17/01/2000]. 
Disponível na Internet:

<http://www.unicamp.br/bc/inform2.htm>

VTLS. Controle de circulação. Trad. Fundação Getúlio Vargas. Divisão de Gestão da Informação. Gerência de Sistemas de Informação. [Rio de Janeiro, RJ] : VTLS/FGV, [1996]. 2p. (folder).

VTLS. VIRTUA system guides : circulation. Version 16.0. Blacksburg, VA : VTLS, 1999.

\section{Agradecimentos}

Os autores apresentam seus especiais agradecimentos aos demais colegas do Grupo de Estudo de Circulação VIRTUA que, apesar de não participarem diretamente da elaboração deste trabalho, participaram das reuniões conforme registrado nas Atas referenciadas. 